\title{
Del aula a la escuela en línea universal: Dimensiones temáticas en canales de Youtube
}

\author{
From the classroom to the universal online school: Thematic dimensions \\ on youtube channels
}

\author{
Arantxa Vizcaíno-Verdú ${ }^{1}$ \\ https://orcid.org/0000-0001-9399-2077 \\ Universidad de Huelva, España \\ Paloma Contreras-Pulido ${ }^{2}$ \\ https://orcid.org/0000-0002-6206-7820 \\ Universidad Internacional de la Rioja, España
}

Recibido: 20-10-2019

Aceptado: 01-12-2019

\section{Cita Recomendada}

Vizcaíno-Verdú, A. \& Contreras-Pulido, P. (2019). Del aula a la escuela en línea universal: Dimensiones temáticas en canales de YouTube. Hamut'ay, 6(3), 12-25.

http://dx.doi.org/10.21503/hamu.v6i3.1841

\section{RESUMEN}

La educación informal en redes sociales es un hecho reciente y creciente en la era de la información ubicua y digital. Un contexto que requiere, por parte del entorno pedagógico institucional, atender a las nuevas dinámicas de enseñanza y aprendizaje, más allá del empleo instrumental de las tecnologías en el aula. Partiendo de esta panorámica mediática actual, el estudio pretende acentuar las dimensiones temáticas educativas referentes y frecuentes en YouTube, así como detectar y plantear nuevos entornos o contenidos didácticos adicionales. Para ello, se parte de un compendio de 196 canales con contenidos generados por instructores académicos o profesionales en su vertiente comercial y/o amateur, desde un diseño metodológico fundamentalmente cualitativo. Se aplica un análisis de contenido temático en la definición de categorías que recogen las materias principales del saber, así como su re-categorización y definición mediante áreas del conocimiento delimitadas en la base de datos bibliográficos de Scopus. Los resultados muestran una frecuencia elevada de contenidos concernientes a las ciencias físicas y sociales. Asimismo, destacan espacios de apariencia dudablemente educativa, cuyo contenido presenta una nueva forma de difundir la cultura general a través de innovadores parámetros comunicativos e informacionales. En conclusión, YouTube se descubre como un medio emergente para la educación, libre de la estructuración formal del aula, proclive a convertirse en la escuela en línea universal.

Palabras Clave: Aprendizaje informal, dimensión temática, YouTube, educación, vídeos generados por instructores.

1 Doctoranda en Comunicación en la Universidad de Huelva (UHU). Máster en Comunicación y Educación Audiovisual (UHU). Graduada en Publicidad y RRPP (UA). Colaboradora de investigación del Grupo Ágora, Grupo Comunicar y la Red Iberoamericana Alfamed Joven. Editora Asociada de la revista Comunicar. arantxavizcaino@gmail.com

2 Doctora en Educación. Profesora de la Universidad Internacional de La Rioja (UNIR) de la Facultad de Educación, del Departamento de Tecnologías Educativas. Miembro del grupo de Investigación Ágora de la Universidad de Huelva y de la Red Iberoamericana en Competencias Mediática Alfamed.paloma.contreras@unir.net 


\begin{abstract}
Informal education on social networking sites is a recent and growing fact in the age of ubiquitous and digital information. It is a context that requires the institutional pedagogical environment to attend the new dynamics of teaching and learning, beyond the instrumental use of technologies in the classroom. Based on this current media scenario, the study aims to emphasize the referential and frequent educational thematic dimensions on YouTube, as well as to detect and propose new environments or additional didactic content. Therefore, we start with a compendium of 196 channels with contents generated by academic or professional instructors in its commercial and/or amateur condition from a fundamentally qualitative methodological design. A thematic content analysis is applied in the definition of categories that include the main subjects of knowledge, as well as their re-categorization and definition through areas of knowledge delimited in the Scopus bibliographic database. The results show a high frequency of content concerning the physical and social sciences. Likewise, spaces of a doubtfully educational nature stand out, whose content presents a new way of disseminating general culture through communicative and informational innovative parameters. In conclusion, YouTube is presented as an emerging medium for education, free from the formal structuring of the classroom, inclined to become the universal online school.
\end{abstract}

Keywords: Informal learning, thematic dimension, YouTube, education, instructor-generated content.

\section{INTRODUCCIÓN}

La era digital, mediática y convergente actual requiere, desde el punto de vista de la educación, ampliar y quebrar las barreras que se extienden en relación a otros modos (y ya no tanto metodologías) de aprender (Camargo, Vieira-Miranda \& Magnoni, 2019). Un mensaje instantáneo vía WhatsApp, una 'historia' pública exclusiva durante 24 horas en Instagram, o un vídeo viralizado en el apartado de tendencias de YouTube son el reflejo de la sociedad actual: información en todos los rincones de Internet y en cada dispositivo (Gómez-del-Castillo, 2017; Picazo-Sánchez, 2016).

En este contexto, se manifiestan nuevos procedimientos de aprendizaje colectivo e informal, que descubren amplitud de posibilidades para producir y recibir información: el contenido generado por instructores (McAlister, 2014). Una tipología esencialmene pedagógica que plantea nuevos interrogantes a las acciones tecnológicas, informacionales y comunicativas desarrolladas en las últimas décadas por la institución escolar.

Desde este panorama, se introduce un estudio exploratorio y cualitativo, cuyo fin pretende acentuar las dimensiones temáticas educativas referentes y frecuentes en YouTube, así como detectar y plantear nuevos entornos o contenidos didácticos ajenos a lo estrictamente formal. Para ello, se planteó como objetivo general subrayar las dimensiones temáticas (áreas del conocimiento) referentes y frecuentes en canales educativos de YouTube y, como objetivo específico, detectar la frecuencia y viabilidad de canales con contenido didáctico ajeno a áreas temáticas preexistentes.

Del aula a la formación reglada en línea: entornos educativos controlados

Desde antaño, la concepción formativa reglada ha generado una suerte de docentes y alumnos que actúan como máquinas evaluadoras y evaluadas, en tanto que el aprendiz o estudiante queda supeditado a un segundo plano (Ausín, Abella, Delgado \& Hortigüela, 2016). Sin embargo, la era tecnológica, informativa y mediática en la que se emprenden actual y necesariamente los procesos didácticos de la escuela formal, conlleva a la apli- 
cación de sistemas de aprendizaje eficaces, activos y significativos. Esto es, emergen alternativas a los redundantes y tradicionales sistemas, como las Tecnologías de la Información y la Comunicación (TIC), los ambientes virtuales de aprendizaje controlado y la tecnología multimedia (Alvites-Huamaní, 2016; Sánchez-Vera, Solano-Fernández \& González-Calatayud, 2016).

En efecto y, tal y como indican López-Belmonte, Pozo-Sánchez, Fuentes-Cabrera \& Trujillo-Torres (2019), los avances tecnológicos de este contexto esencialmente digital e inmersivo se han extendido a todas las áreas de la sociedad, incluida la educación. Un sector que se encuentra en plena renovación, requiriendo una constante transformación de roles y praxis enriquecidas por la tecnología (Arzola, Loya \& González, 2017).

Las escuelas adoptan estrategias innovadoras que, en última instancia, se adaptan a las necesidades y perfiles del alumnado vigente (Area-Moreira, Hernández-Rivero \& Sosa-Alonso, 2016). Esto no significa, empero, que el uso de recursos TIC suponga la sustitución de materiales tradicionales, pues se entienden como un complemento que suscita modelos de aprendizaje híbridos e integradores (Area-Moreira, 2005).

La evolución de las TIC desde la primera década del tercer milenio llegó con grandilocuente terminología, que transcurre del e-learning hasta la Web 2.0, mucho más participativa y social (Fernández-Batanero, Reyes-Rebollo \& Montenegro-Rueda, 2019). Un compendio de didácticas que, por medio de la tecnología y las plataformas digitales, ayuda a desarrollar a los alumnos de todos los estratos educativos habilidades cognitivas, de pensamiento crítico, de acceso, evaluación y síntesis de información, entre otros. En definitiva, aspectos motivacionales que fomentan el interés por los contenidos educativos, y que mejoran la autoestima, independencia y confianza (Chigona \& Chigona, 2011). Alvites-Huamaní (2016) añade que estos entornos en línea facilitan la usabilidad pedagógica en materia de interfaz del usuario, diseño de actividades educativas y verificación y alcance de los objetivos planteados en el aula.

El posicionamiento dicotómico entre docentes a favor y en contra de la implantación de tecno- logías controladas en entornos formales (Pinzón-Capador \& Guerrero-Nieto, 2018), queda relegado a autores como Silva-Pereira, Silva-Roque \& Ferraz-Macário (2018), por la que el uso de recursos tecnológicos en la enseñanza es cada vez más necesario. Se trata, naturalmente, de proporcionar a los alumnos experiencias dinámicas, atractivas y creativas. Incluso la UNESCO, señala Padilla-Partida (2018), considera la formación mediante competencias tecnológicas un procedimiento imprescindible para la institución escolar.

Algunos estudios demuestran el carácter favorable de esta combinación escuela-tecnología y medios: las ventajas de la presencia de las TIC en el entorno universitario (Guerra-Liaño, González-Fernández \& García-Ruiz, 2010); el empleo de redes sociales en entornos educativos (Tuzel \& Hobbs, 2017); la aplicación de metodologías innovadoras en materia de alfabetización digital (Sun, Wang \& Liu, 2017), entre otras experiencias colaborativas y experienciales.

Como apuntan Stephen \& Plowman (2003), la convergencia mediático-digital y la puesta en escena de tecnologías dentro del aula encuentran exitosamente, hoy día, la manera de satisfacer las necesidades de aprendizaje de la sociedad. Se trata, por tanto, de implantar efectiva y eficazmente las TIC como herramientas de enseñanza-aprendizaje, con una perspectiva líquida abierta a nuevos formatos y modos de aprender.

Hacia el aprendizaje informal en redes sociales

En términos de calidad, cantidad, disponibilidad y acceso, hoy el saber está más presente en la sociedad, sobrepasando algunos límites que marcaba la educación formal. Un constante informativo y diario donde Internet se ha convertido en el proveedor pedagógico principal de la era digital. La multiplicidad de canales por el que se percibe y proyecta conocimiento demanda la responsabilidad de la institución escolar, en tanto que su participación resulta fundamental en todos los procesos de aprendizaje existentes (Rodríguez-Gómez, Ion, Mercader \& López-Crespo, 2019).

La ubicuidad y empleo cotidiano de las tecnologías ha dado lugar, como señala Czerkawski (2016), 
a una significativa reestructuración de las experiencias educativas fuera del aula. Como también lo expresan Alvarado-Pazmiño, Ochoa-Mendieta, Ronquillo-Murieta \& Sánchez-Soto (2019), a un medio de aprendizaje que transcurre más allá de la formalidad y el control institucional. En este sentido, González-Sanmamed, Sangrà, Souto-Seijo \& Estévez (2018), afirman que las plataformas y aplicaciones digitales representan un apoyo sustancial para la formación, en tanto en cuanto respetan las necesidades y exigencias actuales. Una metamorfosis didáctica que viene dada por la facilidad de conectar e interactuar con colectivos que comparten intereses comunes, por el empoderamiento progresivo que se deriva del uso tecnológico-digital, y por la admisión de la existencia de un aprendizaje no perceptible y casual (Gozález-Sanmamed, Souto-Seijo, González \& Estévez, 2019).

En este contexto, el aprendizaje formal se refiere a aquella educación impartida en un centro educativo cuyo fin último es la obtención de un certificado. Mientras que el resto de metodologías comprenden el aprendizaje no formal (recibir clases de piano por un profesional en casa) o informal (aprender a cocinar una receta con un vídeo-tutorial) (Galanis, Mayol, Aliert \& García-Peñalvo, 2016). No obstante, y tal y como sugiere Sefton-Green (2013), estas nociones pedagógicas implican la búsqueda activa de fuentes de información por medio de planteamientos auto-dirigidos que, en muchos casos, contrastan con la concepción formal: siempre participa un profesor o mentor, un programa de software educativo y/o un sistema de gestión del aprendizaje. En síntesis, el aprendizaje informal no viene dado por un plan de estudios estructurado, sino por un compendio de recursos disponibles que, por interés o necesidad, quedan a disposición de las personas para consultar y aprender cuándo, dónde y como estimen (Rodríguez-Gómez et al., 2019).

Este formato puede llevarse a cabo por diversas vías, especialmente a través de las redes sociales (Mohmed-Al-Sabaawi \& Mohamed-Dahlan, 2018). Czerkawski (2016) destaca la naturaleza social de estos procedimientos, en tanto que los estudiantes son altamente influenciados por las plataformas digitales: desde el envío instan- táneo de un mensaje, hasta la visita de espacios web, escuchar música, jugar videojuegos y descargar materiales. Estas brindan, en su totalidad, la opción de seleccionar la mejor herramienta para interactuar con otras personas y, al mismo tiempo, auto-gestionar los modos de aprender (Alvarado-Pazmiño et al., 2019).

En este orden de cosas, Greenhow \& Lewin (2016) conceptualizan las redes sociales como prácticas alineadas con el constructivimo y el conectivismo. El primero entiende el aprendizaje como un sistema de participación necesariamente expuesto a contextos colectivos, donde el conocimiento se descentraliza y co-construye con otros. Y el segundo, descubre el procedimiento educativo como una articulación de conexiones en red por medio de nodos y relaciones. Así, el uso de la tecnología digital fuera de la escuela se considera un prometedor vehículo para la formación social, así como para la apertura a modos de vida más flexibles y entretenidos (Pöntinen, Dillon \& Väisänen, 2017).

Hoy las redes sociales están adquiriendo tal popularidad, que no es de extrañar que los estudiantes sean capaces de extrapolar los conocimientos adquiridos en entornos en red a espacios formales y viceversa (Sorte \& Rathod, 2016; Tan, 2013). Así es el caso de YouTube, una red audiovisual de amplio reconocimiento internacional, que ha dado cabida a un ingente número de metodologías de aprendizaje y conocimientos, convirtiéndose en la biblioteca alejandrina del siglo XXI.

El YouTube más educativo: conocimiento universal

YouTube emerge como un emblema de la cultura participativa (Marsh \& Hoff, 2019), jugando un papel principal en la educación por demanda (o aprendizaje informal) a través de prácticas e interacciones sociales como el comentario, la búsqueda o la provisión de información (Dubovi \& Tabak, 2019). En este entorno, surgen los denominados vídeos generados por instructores (D’Aquila, Wang \& Mattia, 2019), que fomentan la producción audiovisual en materia pedagógica con el objetivo de involucrar a los estudiantes en las diferentes temáticas de estudio (Kum-Tien, 
2019). Una metodología que ya algunos autores han ratificado como sistema eficiente para la educación, reportando altos niveles de satisfacción y éxito por parte del alumnado (Kohli, Lancellotti \& Thomas, 2017; Clifton \& Mann, 2011). Draus, Curran \& Trempus (2014) añaden que los estudiantes confían en estos vídeos como medio efectivo de mejora del aprendizaje.

Según Dubovi \& Tabak (2019), los vídeos instructivos de YouTube se clasifican en tres tipos: 1) educación por procedimiento, que resuelve problemas específicos relacionados con componentes motores, visuales, etc. (reemplazo de la memoria de un portátil, ensamblaje de dispositivos, técnicas médicas y musicales, etc.); 2) educación informativa vinculada a ampliar conocimientos generales (lugares para visitar, síntomas de salud, etc.); 3) y educación conceptual-procedimental, enlazada a la expansión de habilidades intelectuales (analizar riesgos, tomar decisiones alternativas, interpretar resultados, etc.). Si bien, la plataforma simplifica estos métodos de enseñanza a dimensiones temáticas que, contrariamente y ante la inmensidad de vídeos disponibles, dificulta la localización de contenidos concretos y necesarios para los estudiantes (Orús et al., 2016; Shoufan, 2018).

Tal es la amplitud de vídeos instructivos en YouTube que, en la actualidad, brotan numerosos estudios sobre su aplicación educativa desde diferentes áreas del conocimiento. Algunos se acogen a la mejora del vocabulario, la gramática, la ortografía o la semántica por medio de blogs (Arndt $\&$ Woore, 2018; Heriyanto, 2015). Otros promueven la lectura fuera del aula (Vizcaíno-Verdú, Contreras-Pulido \& Guzmán-Franco, 2019); señalan la efectividad de YouTube como vía de enseñanza para la química escolar (Bohloko, Makatjane, George \& Mokuku, 2019); conectan música educativa y creaciones audiovisuales en la plataforma (Cayari, 2017), o exploran la identidad musical escolar infantil por medio de vídeos sobre instrumentos de cuerda musicales como el ukelele en YouTube (Smith \& Secoy, 2019); analizan la educación odontológica y la fiabilidad de los contenidos audiovisuales para los estudiantes (DiasDa-Silva, Costa-Pereira \& Walmsley, 2019); profundizan en la participación y compromiso de los alumnos de enfermería por medio de vídeos educativos (Johnston, Barton, Williams-Pritchard \& Todorovic, 2017); observan la fiabilidad y valor educacional de los vídeos publicados sobre cirugía (Toolabi, Parsaei, Elyasinia \& Zamanian, 2019) y la calidad informacional de estos para la especialización inmunológica en estudiantes de medicina (Van-den-Eynde et al., 2019).

En suma, un extracto representativo y actualizado que deja entrever la infinidad de posibilidades educativas de YouTube, y el creciente y exponencial número de vídeos dedicados a las diferentes áreas del conocimiento, donde instructores profesionales y/o profesores de la institución formal proyectan sus saberes para la comunidad global. Desde este punto, se parte hacia la concreción de las dimensiones temáticas en la plataforma, y a la frecuencia de las mismas en los canales con mayor impacto educativo de la actualidad.

\section{Materiales y Métodos}

\section{Participantes}

La unidad de muestreo para el análisis de contenido temático se ha delimitado por medio de la técnica no probabilística por conveniencia, dada la amplitud y divergencia de canales publicados en YouTube. Para ello, se han seleccionado dos rankings de canales educativos de 2019 de Feedspot (categoría 'Educational YouTube Channels on Learning, Discovery \& Educational Videos' y 'Teacher Youtube Channels on Educational Videos, Tutorials, Lessons \& Courses'), que recopilan espacios por estratos destacados en base a la relevancia, seguidores y datos estadísticos proporcionados por Alexa Web Traffic Rank. La razón por la que se escogieron estas clasificaciones se fundamenta en la compilación de canales educativos de alto impacto, medidos por sistemas algorítmicos de tendencia.

En suma, se observaron un total de 196 canales de YouTube adscritos al área de educación (en su vertiente comercial y amateur). Puesto que algunos de ellos coincidían en ambas clasificaciones, fueron unificados en un mismo listado (Tabla 1). 
Tabla 1

Unidad de muestreo y unidad de registro para el análisis

\begin{tabular}{lcl}
\hline \multicolumn{1}{c}{ Unidad de muestreo } & $\begin{array}{c}\text { Número } \\
\text { de cana- } \\
\text { les }\end{array}$ & $\begin{array}{c}\text { Unidad de } \\
\text { registro }\end{array}$ \\
\hline $\begin{array}{l}\text { Ranking 'Top educational } \\
\text { YouTube channels on learning, }\end{array}$ & 196 & $\begin{array}{l}\text { Descripción, } \\
\text { título del } \\
\text { canal y de } \\
\text { discovery \& educational videos' }\end{array}$ \\
$\begin{array}{l}\text { Feedspot (https://bit.ly/2qJPios). } \\
\text { Ranking 'Top teacher YouTube } \\
\text { channels on educational videos, } \\
\text { tutorials, lessons \& courses' }\end{array}$ & & $\begin{array}{l}\text { vídeos } \\
\text { publicados }\end{array}$ \\
Feedspot (https://bit.ly/32TOE- & & \\
TE) & & \\
\hline
\end{tabular}

Fuente: Elaboración propia (2019).

La parte de la unidad analizable para la detección de temáticas se refiere, concretamente, a la de cada canal (unidad de registro), obteniendo la temática aproximada inicial a partir de la descripción disponible (sección 'más información'), el título del canal y el título de los últimos vídeos publicados.

\section{Instrumento}

El procedimiento se ejecutó a través de una rejilla de observación adaptada al objeto de estudio, a partir de la concepción de Díaz-Herrera (2018) (Tabla 2).

\section{Tabla 2}

Instrumento de análisis de contenido temático

\begin{tabular}{|c|c|c|c|c|}
\hline Canal & Título & $\begin{array}{l}\text { Descrip- } \\
\text { ción }\end{array}$ & $\begin{array}{l}\text { Temática(s) } \\
\text { inductivas }\end{array}$ & Observaciones \\
\hline $\begin{array}{l}\text { Enla- } \\
\text { ce }\end{array}$ & $\begin{array}{l}\text { Título } \\
\text { del } \\
\text { canal }\end{array}$ & $\begin{array}{l}\text { Des- } \\
\text { cripción } \\
\text { recogida } \\
\text { en la } \\
\text { sección } \\
\text { 'más } \\
\text { informa- } \\
\text { ción' }\end{array}$ & $\begin{array}{l}\text { Descrip- } \\
\text { ción termi- } \\
\text { nológica } \\
\text { de uno a } \\
\text { tres tér- } \\
\text { minos de } \\
\text { temática }\end{array}$ & $\begin{array}{l}\text { Pertenencia } \\
\text { a un medio } \\
\text { profesional/ } \\
\text { comercial, dis- } \\
\text { ponibilidad del } \\
\text { contenido o } \\
\text { ampliación de } \\
\text { la información } \\
\text { si no se re- } \\
\text { gistra en una } \\
\text { única temática } \\
\text { (curiosidades) }\end{array}$ \\
\hline
\end{tabular}

Fuente: Elaboración propia (2019).

La configuración instrumental descendente aplicada a la muestra de 196 canales educativos en YouTube, posibilitó la detección recurrente de temáticas emergentes en cada canal, así como la frecuencia en cada uno de ellos. De este modo y, atendiendo a la base de datos bibliográfica internacional Scopus, se definieron las áreas temáticas que descubren la producción científica mundial, a fin de comparar campos de conocimiento en tendencia en YouTube y campos de conocimiento científico formales (Tabla 3).

Tabla 3

Campos temáticos estandarizados en Scopus

\begin{tabular}{|c|c|c|}
\hline $\begin{array}{l}\text { Unidad de } \\
\text { muestreo }\end{array}$ & $\begin{array}{l}\text { Número de } \\
\text { canales }\end{array}$ & Unidad de registro \\
\hline \multirow[t]{4}{*}{ Áreas Scopus } & $\begin{array}{l}\text { Ciencias de } \\
\text { la salud }\end{array}$ & $\begin{array}{l}\text { Medicina, } \\
\text { Enfermería, } \\
\text { Veterinaria, } \\
\text { Odontología, } \\
\text { Profesiones de la salud }\end{array}$ \\
\hline & $\begin{array}{l}\text { Ciencias de } \\
\text { la vida }\end{array}$ & $\begin{array}{l}\text { Ciencias de la agricultura } \\
\text { y la biología; } \\
\text { Bioquímica, genética y } \\
\text { biología molecular; } \\
\text { Inmunología y microbio- } \\
\text { logía; } \\
\text { Neurociencia; } \\
\text { Farmacología, toxicología } \\
\text { y farmacéutica }\end{array}$ \\
\hline & $\begin{array}{l}\text { Ciencias } \\
\text { físicas }\end{array}$ & $\begin{array}{l}\text { Ingeniería química, Qími- } \\
\text { ca Ciencias informáticas, } \\
\text { Ciencias de la tierra y } \\
\text { planetarias, Energías, } \\
\text { Ingeniería, Ciencia } \\
\text { medioambiental, Ciencias } \\
\text { materiales,Matemáticas, } \\
\text { Física y astronomía }\end{array}$ \\
\hline & $\begin{array}{l}\text { Ciencias } \\
\text { sociales }\end{array}$ & $\begin{array}{l}\text { Artes y humanidades; } \\
\text { Negocios, gestión y } \\
\text { contabilidad; Ciencias de } \\
\text { la decisión; Economía, } \\
\text { Econometría y Finanzas; } \\
\text { Psicología; Ciencias } \\
\text { sociales multidisciplinar }\end{array}$ \\
\hline
\end{tabular}

Fuente: Elaboración propia a partir de Scopus (2019).

\section{Tipo y diseño de estudio}

La metodología se circunscribe al procedimiento deductivo de Arbeláez \& Onrubia (2014), por el que se parte de una fase teórica y de pre-análisis de la información a una fase descriptivo-analítica e interpretativa de categorías emergentes. En esta línea y, puesto que se pretende desarrollar un planteamiento lógico-cualitativo y exploratorio mediante análisis de contenido temático (Díaz-Herrera, 2018), no se proponen hipótesis. El propósito último es, entonces, sistematizar ten- 
dencias educativas en canales de YouTube, acotarlas a áreas científicas internacionalmente reconocidas y/o extraer nuevas estrategias de aprendizaje no contempladas previamente.

\section{Procedimiento}

La fórmula empleada para el análisis de contenido temático queda definida por una primera fase de construcción de unidades comunes. En ella y, tal y como se replica en la Tabla 4, se parte de: 1) sistematización de temas; 2) configuración de categorías; 3) creación de un árbol categórico; 4) validación de categorías mediante conglomerados adyacentes; y 5) definición de temáticas centrales.

\section{Tabla 4}

Fase inductiva de la primera fase

\begin{tabular}{ll}
\hline \multicolumn{1}{c}{$\begin{array}{c}\text { Unidad de } \\
\text { muestreo }\end{array}$} & \multicolumn{1}{c}{ Unidad de registro } \\
\hline $\begin{array}{l}\text { Sistematiza- } \\
\text { ción de temas }\end{array}$ & $\begin{array}{l}\text { Generar listado con título, descripción y } \\
\text { observación de cada canal. }\end{array}$ \\
\hline $\begin{array}{l}\text { Configuración } \\
\text { de categorías }\end{array}$ & $\begin{array}{l}\text { Establecer unidades de registros elemen- } \\
\text { tales y significativas mediante proceso } \\
\text { inferencial inductivo e identificar frecuen- } \\
\text { cias temáticas recurrentes. }\end{array}$ \\
\hline $\begin{array}{l}\text { Creación árbol } \\
\text { de categorías }\end{array}$ & $\begin{array}{l}\text { Reestructuración de categorías por fami- } \\
\text { lias conceptuales. }\end{array}$ \\
\hline $\begin{array}{l}\text { Validación de } \\
\text { categorías }\end{array}$ & $\begin{array}{l}\text { Confección y re-categorización de temáti- } \\
\text { cas por conglomerados adyacentes. }\end{array}$ \\
\hline Temática & $\begin{array}{l}\text { Producto final con etiquetas/dimensiones } \\
\text { temáticas centrales y frecuencia de cana- }\end{array}$ \\
central & les (Scopus). \\
\hline
\end{tabular}

Fuente: Elaboración propia a partir de Díaz-Herrera (2018).

La segunda fase queda suscrita a la comparativa entre el listado de temáticas centrales en YouTube y los campos del conocimiento reglados por Scopus. Esta actuación permitirá destacar dimensiones temáticas recurrentes en los canales, así como revelar nuevos sectores educativos en alza en entornos digitales de aprendizaje informal.

\section{Resultados}

Para el análisis de los resultados se llevó a cabo la observación de los 196 canales educativos en YouTube, con el fin de extraer temáticas (materias) indispensablemente pedagógicas. De la primera fase, se extrajo un listado que recogía enlace directo al canal, título del canal, descripción de la sección 'más información', la temática inicial observada de modo inductivo y observaciones mínimas requeridas (ver base de datos completa en https:// doi.org/10.6084/m9.figshare.10063028.v1).

Seguidamente y, mediante la configuración categórica, se definieron etiquetas educativas significativas según frecuencia de apariciones y porcentaje equivalente. De este compendio, se recopilaron una totalidad de 59 temáticas iniciales (ver anexos), donde sobresalieron en orden descendente las dimensiones: a) curiosidades (con contenidos múltiples sin materia global definida) (18\%); b) física (17\%); c) matemáticas (16\%); d) lengua inglesa (9\%); e) historia (4\%); f) biología (4\%); g) química (3\%); h) astronomía (3\%); i) informática $(2 \%)$. El resto de etiquetas quedaron excluidas al mínimo o nulo valor porcentual.

A continuación, se re-categorizaron las etiquetas iniciales por área temática, de modo que se agruparon materias adyacentes para establecer y validar un árbol categórico del conocimiento. A partir de estos se establecieron las dimensiones temáticas definitivas acogidas por la base de datos bibliográfica Scopus. En la Tabla 5 se muestra la frecuencia resultante de dimensiones temáticas educativas en YouTube.

\section{Tabla 5}

Re-categorización, validación y definición de dimensiones temáticas educativas en YouTube

\begin{tabular}{lllll}
\hline $\begin{array}{l}\text { Re-categoriza- } \\
\text { ción temáticas } \\
\text { iniciales por área }\end{array}$ & $\begin{array}{l}\text { Árbol } \\
\text { categórico }\end{array}$ & $\begin{array}{l}\text { Categoría } \\
\text { definitiva } \\
\text { (Scopus) }\end{array}$ & $\begin{array}{l}\text { Frecuen- } \\
\text { cia temá- } \\
\text { tica en } \\
\text { canales }\end{array}$ & $\begin{array}{l}\text { equi- } \\
\text { va- } \\
\text { lente }\end{array}$ \\
\hline Física, Química & $\begin{array}{l}\text { Ciencias } \\
\text { físicas, }\end{array}$ & $\begin{array}{l}\text { Ciencias } \\
\text { físicas }\end{array}$ & 202 & 45 \\
Matemáticas, & químicas, & & \\
Ciencias & & & \\
informáticas y & matemáti- & & \\
TIC, Tecnología & cas, infor- & & \\
y electrónica, & máticas y & & \\
Astronomía, & tecnológi- & & \\
Ingenierías, & cas & & \\
Robótica e & & & \\
inteligencia & & & \\
artificial & & &
\end{tabular}




\begin{tabular}{|c|c|c|c|c|}
\hline $\begin{array}{l}\text { Re-categoriza- } \\
\text { ción temáticas } \\
\text { iniciales por área }\end{array}$ & $\begin{array}{l}\text { Árbol } \\
\text { categórico }\end{array}$ & $\begin{array}{l}\text { Categoría } \\
\text { definitiva } \\
\text { (Scopus) }\end{array}$ & $\begin{array}{l}\text { Frecuen- } \\
\text { cia temá- } \\
\text { tica en } \\
\text { canales }\end{array}$ & $\begin{array}{l}\% \\
\text { equi- } \\
\text { va- } \\
\text { lente }\end{array}$ \\
\hline $\begin{array}{l}\text { Lengua, } \\
\text { literatura y } \\
\text { filología, Arte y } \\
\text { humanidades, } \\
\text { Economía } \\
\text { y finanzas, } \\
\text { Ciencias } \\
\text { del deporte, } \\
\text { Filosofía y teo- } \\
\text { logía, Turismo, } \\
\text { Educación y } \\
\text { pedagogía, } \\
\text { Filosofía, } \\
\text { Ciencias polí- } \\
\text { ticas, Ciencias } \\
\text { medioambien- } \\
\text { tales }\end{array}$ & $\begin{array}{l}\text { Ciencias } \\
\text { sociales, } \\
\text { jurídicas, } \\
\text { humani- } \\
\text { dades y } \\
\text { artes }\end{array}$ & $\begin{array}{l}\text { Ciencias } \\
\text { sociales }\end{array}$ & 110 & 25 \\
\hline $\begin{array}{l}\text { Curiosidades, } \\
\text { Videojuegos, } \\
\text { Astrología }\end{array}$ & Otros & Otros & 83 & 19 \\
\hline $\begin{array}{l}\text { Ciencias } \\
\text { farmacológi- } \\
\text { cas, Ciencias } \\
\text { biológicas } \\
\text { y genética, } \\
\text { Agricultura y } \\
\text { ecología, Flora } \\
\text { y fauna }\end{array}$ & $\begin{array}{l}\text { Ciencias } \\
\text { biológi- } \\
\text { cas, de la } \\
\text { naturaleza } \\
\text { y la vida }\end{array}$ & $\begin{array}{l}\text { Ciencias } \\
\text { de la } \\
\text { vida }\end{array}$ & 33 & 7 \\
\hline $\begin{array}{l}\text { Medicina, } \\
\text { Enfermería, } \\
\text { Odontología, } \\
\text { Nutrición }\end{array}$ & $\begin{array}{l}\text { Ciencias } \\
\text { de la } \\
\text { salud }\end{array}$ & $\begin{array}{l}\text { Ciencias } \\
\text { de la } \\
\text { salud }\end{array}$ & 16 & 4 \\
\hline
\end{tabular}

Fuente: Elaboración propia (2019).

La dimensión más destacada en la plataforma audiovisual comprende el área de Ciencias físicas ( $45 \%$ del total), seguida de las Ciencias Sociales (25\%), otros (19\%), Ciencias de la vida (7\%) y Ciencias de la salud (4\%). Dado que la categoría 'otros' predominaba respecto de dos dimensiones académico-científicas preestablecidas, se compilaron aleatoriamente uno de los últimos títulos publicados en cada uno de estos canales, con la finalidad de identificar similitudes en la tipología de contenido y planteamiento expositivo (Tabla 6).
Tabla 6

Títulos canales 'otros'

\begin{tabular}{|c|c|}
\hline $\begin{array}{l}\text { Número de } \\
\text { canales }\end{array}$ & Unidad de registro \\
\hline $\begin{array}{l}\text { SmarterE- } \\
\text { veryDay }\end{array}$ & $\begin{array}{l}\text { How to Plant } 20 \text { MILLION TREES - Smarter } \\
\text { Every Day } 227\end{array}$ \\
\hline Vsauce & ¿Qué es lo más aterrador? \\
\hline $\begin{array}{l}\text { AsapSCIEN- } \\
\text { CE }\end{array}$ & How To Fall Asleep In 2 Minutes \\
\hline $\begin{array}{l}\text { Kurzgesagt - } \\
\text { In a Nutshell }\end{array}$ & $\begin{array}{l}\text { ¿Es la carne mala para ti? ¿Es la carne poco } \\
\text { saludable? }\end{array}$ \\
\hline Vox & Is the gray wolf actually endangered? \\
\hline CGP Grey & $\begin{array}{l}\text { ¿Quién es dueño de la Estatua de la Liber- } \\
\text { tad? (Nueva Jersey vs. Nueva York) }\end{array}$ \\
\hline Vlogbrothers & Why Are Poor Countries Poor? \\
\hline RealLifeLore & $\begin{array}{l}\text { What's the Longest Walk-able Distance on } \\
\text { Earth? }\end{array}$ \\
\hline $\begin{array}{l}\text { It's Okay To } \\
\text { Be Smart }\end{array}$ & 7 Scientific Urban Legends Debunked! \\
\hline $\begin{array}{l}\text { Science } \\
\text { Channel }\end{array}$ & How Belts Are Made | How It's Made \\
\hline Big Think & $\begin{array}{l}\text { How does stress affect a child's development } \\
\text { and academic potential? | Pamela Cantor }\end{array}$ \\
\hline list25 & 25 Super Cool Facts About Jupiter \\
\hline MinuteEarth & Why Are Adults Bad At New Languages? \\
\hline $\begin{array}{l}\text { Today I } \\
\text { Found Out }\end{array}$ & Why Is There No E in the A-F Grading Scale? \\
\hline Mental Floss & $\begin{array}{l}\text { Everything You Need to Know About Tourism } \\
\text { - Mental Floss Scatterbrained }\end{array}$ \\
\hline Stanford & Solving for Energy | Sally Benson \\
\hline $\begin{array}{l}\text { BBC Earth } \\
\text { Lab }\end{array}$ & $\begin{array}{l}\text { How Binge Drinking Effects Your Blood } \\
\text { Vessels | Horizon | Earth Lab }\end{array}$ \\
\hline THNKR & $\begin{array}{l}\text { 1st Newspaper Print of Declaration of Inde- } \\
\text { pendence }\end{array}$ \\
\hline $\begin{array}{l}\text { IncredibleS- } \\
\text { cience }\end{array}$ & $\begin{array}{l}\text { How To Make Fire From Water DIY Incredi- } \\
\text { ble Science Experiment }\end{array}$ \\
\hline $\begin{array}{l}\text { HowStu- } \\
\text { ffWorks }\end{array}$ & Can 3-D Printing Help People Walk? \\
\hline Sick Science! & $\begin{array}{l}10 \text { Amazing Egg Experiments - Steve Span- } \\
\text { gler Science }\end{array}$ \\
\hline Joe Scott & $\begin{array}{l}\text { How We Could Survive On Mars - Feat. Andy } \\
\text { Weir | Answers With Joe }\end{array}$ \\
\hline $\begin{array}{l}\text { Thebrains- } \\
\text { coop }\end{array}$ & How Birds inspire Builders \\
\hline $\begin{array}{l}\text { Gross } \\
\text { Science }\end{array}$ & Why Does This Frog Have So Many Legs?! \\
\hline $\begin{array}{l}\text { SpanglerS- } \\
\text { cienceTV }\end{array}$ & $\begin{array}{l}\text { Exploding Potato Chips - Cool Science Demo } \\
\text { with Pringles }\end{array}$ \\
\hline New Scientist & $\begin{array}{l}\text { These sharks use unique molecules to glow } \\
\text { green }\end{array}$ \\
\hline Thought Café & Autism: Think Differently \\
\hline
\end{tabular}




\begin{tabular}{|c|c|}
\hline $\begin{array}{l}\text { Número de } \\
\text { canales }\end{array}$ & Unidad de registro \\
\hline $\begin{array}{l}\text { Above The } \\
\text { Noise }\end{array}$ & Are Zoos Good or Bad for Animals? \\
\hline $\begin{array}{l}\text { TED-Ed - } \\
\text { Lessons Wor- } \\
\text { th Sharing }\end{array}$ & The Maya myth of the morning star \\
\hline $\begin{array}{l}\text { Teacher's } \\
\text { Tech }\end{array}$ & $\begin{array}{l}\text { Top } 6 \text { FREE Video Editors for YOU to try! } \\
\text { (Easy to Advanced) }\end{array}$ \\
\hline The Teacher & $\begin{array}{l}\text { How To Use Clipboard in Windows } 10 \text { and } \\
\text { Microsoft Office } 2019 \text { Tutorial }\end{array}$ \\
\hline $\begin{array}{l}\text { Teaching } \\
\text { Channel }\end{array}$ & $\begin{array}{l}\text { How to Use the Tch Plus Recorder App, iOS } \\
\text { Version }\end{array}$ \\
\hline $\begin{array}{l}\text { Kayse Morris } \\
\text { - Teaching on } \\
\text { Less }\end{array}$ & $\begin{array}{l}\text { How Teachers Can Sell \& Market Their } \\
\text { Resources Online }\end{array}$ \\
\hline $\begin{array}{l}\text { Jennuine } \\
\text { Teaching }\end{array}$ & $\begin{array}{l}\text { How I Teach Informational Writing ||Part } \\
\text { One\| }\end{array}$ \\
\hline $\begin{array}{l}\text { One FAB } \\
\text { Teacher }\end{array}$ & $\begin{array}{l}\text { Classroom Management: Whole Group } \\
\text { Behavior Incentives }\end{array}$ \\
\hline $\begin{array}{l}\text { Teach For } \\
\text { America }\end{array}$ & Why This Work Is So Important \\
\hline $\begin{array}{l}\text { The Tea- } \\
\text { cher's Library }\end{array}$ & $\begin{array}{l}\text { She Persisted: } 13 \text { American Women Who } \\
\text { Changed the World }\end{array}$ \\
\hline $\begin{array}{l}\text { Kate The } \\
\text { Sleepy } \\
\text { Teacher }\end{array}$ & $\begin{array}{l}\text { CLASSROOM TOUR I ELEMENTARY I } 2019 \\
\text { - } 2020 \text { I BEHIND THE SCENES CLASS- } \\
\text { ROOM REVEAL! }\end{array}$ \\
\hline
\end{tabular}

Fuente: Elaboración propia (2019).

Del listado se distinguieron rasgos comunes en materia de contenido y concepción lingüística titular. De manera que: 1) se emplean esencialmente preguntas informativas (o wh- questions en el habla anglosajona), donde destacan patrones como el 'how to' y 'why'); 2) apelaciones al conocimiento (conoce...; descubre...); 3) rankings y clasificaciones ('top', '20 curiosidades...'); 4) experimentos ('resolvemos'..., 'explotamos...'); y 5) contenidos variados, dispares y sin área de conocimiento científica dada. En este último caso, se observa información que, en muchos casos, expone datos ajenos, conjeturas, teorías de la conspiración y cultura general.

\section{Discusión y Conclusiones}

El aprendizaje informal y, concretamente, la producción y consumo de conocimientos en redes sociales, es un hecho constatable en la actualidad, tal y como presentan estudios precedentes en YouTube (Arndt \& Woore, 2018; Bohloko, Makatjane, George \& Mokuku, 2019; Cayari, 2017; Heriyanto, 2015; Vizcaíno-Verdú et al., 2019). Los enfoques y temáticas son múltiples, en muchos casos debido a las tendencias en la Red, y en otros, debido a las exigencias informativas de los usuarios.

La presente investigación, en su carácter exploratorio, trata de evidenciar la apertura de esta plataforma audiovisual y social a la comunidad educativa, ya sean académicos o profesionales de un área científica o técnica concreta. $Y$ es que, ante la multiplicidad de vídeos educativos publicados en YouTube, encontramos en una mínima muestra, un vehemente interés por las denominadas ciencias duras (ciencias físicas), seguidas de las ciencias blandas (ciencias sociales) y el contenido misceláneo. Tres categorías que, fuera de lo estrictamente formal, plantean contenidos adicionales al aula, exponiendo las ventajas de la red social como medio pedagógico disponible para profesores y alumnos (Alvarado-Pazmiño et al., 2019; Pöntinen et al., 2017). En este sentido, damos cuenta de la trayectoria educativa y multidisciplinar de YouTube, así como del interés de las ciencias académico-científicas por contribuir con el saber más allá del entorno institucional.

Se trata, de este modo, de ofrecer información desinteresada que, fuera de la estructura formal de la escuela y las posibilidades que otorgan las TIC, es percibida, compartida y empleada por toda la comunidad internauta (Rodríguez-Gómez et al., 2019). Ciertamente, no hay límite de edad ni criterios taxativos que obstruyan el acceso a la educación en YouTube. Si bien, el filtro viene a anexionarse por otras vías no contempladas en el análisis, aunque igualmente implicadas: la brecha digital, la desinformación, la valoración y actitud crítica ante el ingente número de horas de vídeo publicadas cada hora (Alonso-González, 2019; Carbonell-Alcocer \& Gertrúdix-Barrio, 2019; Vargas-Vergara, 2019), entre otras.

Cabe hacer especial mención de la amplia variedad de producciones recopiladas en la categoría 'otros', donde distinguimos una línea de divulgación esencialmente científica (más que educativa), que describe un formato ajeno a las líneas 
temáticas reconocidas. Es decir, los contenidos de estos canales difieren unos de otros, evidenciando (y dificultando), la asignación de una categoría global. Los últimos vídeos publicados en esta sección reflejan un formato propio del clickbait (cebo), por el que, mediante el título, se pretende atraer la atención de usuarios para que accedan y visualicen el contenido (Chen, Conroy \& Rubin, 2015). Pero, ¿por qué se incorporan dichos canales como medio educativo? ¿Qué los diferencia del canal de una profesora en física o un profesor en inglés? Se habría de incidir, en este sentido, en el análisis del ranking consultado, así como en los parámetros que construyen o caracterizan estos canales. ¿La temática, en general, da prioridad a todas las ciencias? ¿Se basan en información y fuentes contrastadas científica y académicamente? Estas cuestiones, además, inducen al replanteamiento del estudio desde la visión del alumno/ usuario. Desde esta perspectiva, se plantea ampliar el análisis con una muestra de seguidores en cada categoría (ciencias físicas, ciencias sociales, otros, etc.), con el fin de observar y delimitar el perfil de los canales educativos y divulgativos. Es más, esta proposición sugiere un trabajo que entrecruce si, realmente, los canales educativos son gestionados por profesores/instructores/científicos, y si los seguidores que reciben la información son alumnos de dichas materias o, sencillamente, usuarios esporádicos.

Ante este panorama y, conscientes del carácter iniciático del estudio, podemos intuir nuevas líneas de investigación en cuanto a análisis educativos en la plataforma se refieren. Entre ellos, se recomienda profundizar en las razones por las que se observa un mayor conglomerado de productos científicos físicos, y no tanto sociales y humanísticos en YouTube (y si esto se reproduce a una cobertura mayor). Al mismo tiempo, se propone analizar el fenómeno del contenido misceláneo o los canales (supuestamente) educativos, cuyos datos e informaciones recogen vídeos variopintos y errantes entre la validez académica y el mero entretenimiento conjetural. En conjunto, se propone desarrollar una revisión más amplia sobre la réplica de dimensiones temáticas en otros entornos geográficos, las características comunes entre las diferentes categorías académicas, la validez y fiabilidad de los contenidos generados por los ins- tructores en YouTube, el perfil de la comunidad de estos canales, y las razones por las que estos espacios didácticos emergentes adquieren tanta relevancia en la sociedad.

\section{Agradecimiento}

Este trabajo se enmarca en 'Alfamed' (Red Euroamericana de Investigadores), con el apoyo del Proyecto I+D "YOUTUBERS E INSTAGRAMMERS: LA COMPETENCIA MEDIÁTICA EN LOS PROSUMIDORES EMERGENTES” (RTI2018-093303-B-I00), financiado por la Agencia Estatal de Investigación del Ministerio de Ciencia, Innovación y Universidades de Espańa y el Fondo Europeo de Desarrollo Regional (FEDER).

\section{REFERENCIAS BIBLIOGRÁFICAS}

Alvarado-Pazmińo, E. R., Ochoa-Mendieta, M. A., Ronquillo-Murieta, G. V., \& Sánchez-Soto, M. A. (2019). Importancia y uso de las redes sociales en la educación. Mundo de la Investigación y el Conocimiento, 3(2), 882-893. https:// doi.org/10.26820/recimundo/3.(2).abril.2019.882-893

Alonso-González, M. (2019). Fake news: Desinformación en la era de la sociedad de la información. Ámbitos, 45, 2952. https://doi.org/10.12795/Ambitos.2019.i45.03

Alvites-Huamaní, P. A. (2016). Usabilidad: Páginas web, entornos y educación virtual, 3(1), 71-79. https://doi. org/10.21503/hamu.v3i1.1002

Arbeláez, M., \& Onrubia, J. (2014). Análisis bibliométrico y de contenido. Dos metodologías complementarias para el análisis de la revista colombiana Educación y Cultura. Revista de investigaciones UCM, 14(23), 14-31. https://doi. org/10.22383/ri.v14i1.5

Area-Moreira, M. (2005). The information and communication technologies in the school system. A review of the research lines. Relieve, 11(1), 3-25. Recuperado de https:// bit.ly/341nfPE

Area-Moreira, M., Hernández-Rivero, V., \& Sosa-Alonso, J. J. (2016). Modelos de integración didáctica de las TIC en el aula. Comunicar, 47, 79-87. https://doi.org/10.3916/ C47-2016-08

Arndt, H. L., \& Woore, R. (2018). Vocabulary learning from watching YouTube videos and reading blog posts. Language Learning \& Technology, 22(3), 124-142. https://doi. org/10125/44660

Arzola, D., Loya, C. \& González, A. (2017). El trabajo di- 
rectivo en Educación Primaria: Liderazgo, procesos participativos y democracia escolar. Revista de Investigación Educativa de la REDIECH, 7(2), 35-41. Recuperado de https:// bit.ly/2PgWjr7

Ausín, V., Abella, V., Delgado, V., \& Hortigüela, D. (2016). Aprendizaje basado en proyectos a través de las TIC. Una experiencia de innovación docente desde las aulas universitarias. Formación Universitaria, 9(3). https://doi.org/10.4067/ S0718-50062016000300005

Bohloko, M., Makatjane, T. J., George, M. J., \& Mokuku, T. (2019). Assessing the effectiveness of using YouTube videos in teaching the chemistry of group I and VII elementos in a High School in Lesotho. African Journal of Research in Mathematics, Science and Technology Education, 23(1), 75-85. https://doi.org/10.1080/18117295.2019.1593610

Camargo, A. C., Vieira-Miranda, G., \& Magnoni, A. F. (2019). Convergência midiática e cultura participativa: A possibilidade de novas relaçóes entre os agentes sociais no campo da comunicação e as novas tecnologias. Disertaciones, 12(1), 75-87. https://doi.org/10.12804/revistas.urosario.edu.co/disertaciones/a.6071

Carbonell-Alcocer, A., \& Gertrúdix-Barrio, M. (2019). Evaluación de una intervención educativa para la alfabetización en datos mediante el uso del método científico y el aprendizaje situado. Revista Mediterránea de Comunicación, 10(2), 213-241. https://doi.org/10.14198/MEDCOM2019.10.2.6

Cayari, C. (2017). Connecting music education and virtual performance practices from YouTube. Music Education Research, 20(3), 360-376. https://doi.org/10.1080/14613808 .2017 .1383374

Chen, Y., Conroy, N. J., \& Rubin, V. L. (2015). Misleading online content: Recognizing clickbait as 'false news'. In Proceedings of the 2015 ACM on Workshop on Multimodal Deception Detection (pp. 15-19). Seattle: ACM. https:// doi.org/10.1145/2823465.2823467

Chigona, A., \& Chigona, W. (2011). An investigation of factors affecting the use of ICT for teaching in the Western cape schools. In 18th European Conference on Information Systems (pp. 1-12). South Africa: University of Pretoria. Recuperado de https://bit.ly/36ctF0r

Clifton, A., \& Mann, C. (2011). Can YouTube enhance student nurse learning? Nurse Education Today, 31, 311-313. https://doi.org/10.1016/j.nedt.2010.10.004

Czerkawski, B. C. (2016). Blending formal and informal learning networks for online learning. International Review of Research in Open and Distributed Learning, 17(3), 138156. https://doi.org/10.19173/irrodl.v17i3.2344

D’Aquila, J. M., Wang, D., \& Mattia, A. (2019). Are instructor generated YouTube videos effective in accounting classes? A study of student performance, engagement, motivation, and perception. Journal of Accounting Education, 47, 63-74. https://doi.org/10.1016/j.jaccedu.2019.02.002

Dias-da-Silva, M. A., Costa-Pereira, A., \& Walmsley, A. D. (2019). Who is providing dental education content via You-
Tube? British Dental Journal, 226(6), 437-440. https:/doi. org/10.1038/s41415-019-0046-8

Díaz-Herrera, C. (2018). Investigación cualitativa y análisis de contenido temático. Orientación intelectual de revista Universum. Revista General de Información y Documentación, 28(1), 119-142. https://doi.org/10.5209/ RGID.60813

Draus, P., Curran, M., \& Trempus, M. (2014). The influence of instructor-generated video concent on student satisfaction with and engagement in asynchronous online classes. Journal of Online Learning and Teaching, 10(2), 240-254. Recuperado de https://bit.ly/2BPekEM

Dubovi, I., \& Tabak, I. (2019). Examining the educative value of person-to-person knowledge sharing on social media: The case of YouTube as a site of CSCL? In 13th International Conference on Computer Supported Collaborative Learning (pp. 593-596). CSCL: France. Recuperado de https://bit.ly/2NeDFNO

Fernández-Batanero, J. M., Reyes-Rebollo, M. M., \& Montenegro-Rueda, M. (2019). Impact of ICT on students with high abilities. Bibliographic review (2008-2018). Computers \& Education, 137, 48-58. https://doi.org/10.1016/j. compedu.2019.04.007

Galanis, N., Mayol, E., Alier, M., \& García-Peñalvo, F. J. (2016). Supporting, evaluation and validating informal learning. A social approach. Computers in Human Behavior, 55, 596-603. https://doi.org/10.1016/j.chb.2015.08.005

Gómez-del-Castillo, M. T. (2017). Utilización de WhatsApp para la comunicación en titulados superiores. REICE, 15(4), 51-65. https://doi.org/10.15366/reice2017.15.4.003

González-Sanmamed, M., Sangrà, A., Souto-Seijo, A., \& Estévez, I. (2018). Ecologías de aprendizaje en la era digital: Desafíos para la Educación Superior. Publicaciones, 48, 11 38. https://doi.org/10.30827/publicaciones.v48i1.7329

González-Sanmamed, M., Souto-Seijo, A., González, I., \& Estévez, I. (2019). Aprendizaje informal y desarrollo profesional: Análisis de las ecologías de aprendizaje del profesorado en Educación Infantil. EDUTEC, 68, 70-81. https:// doi.org/10.21556/edutec.2019.68.1305

Guerra-Liaño, S., González-Fernández, N., \& García-Ruiz, R. (2010). Utilización de las TIC por el profesorado universitario como recurso didáctico. Comunicar, 35, 141-147. https://doi.org/10.3916/C35-2010-03-07

Greenhow, C., \& Lewin, C. (2016). Social media and education: Reconceptualizing the boundaries of formal and informal learning. Learning, Media and Technology, 41, 6-30. https://doi.org/10.1080/17439884.2015.1064954

Heriyanto, D. (2015). The effectiveness of using YouTube for vocabulary mastery. Eternal, 6(1), 1-12. https://doi. org/10.26877/eternal.v6i1.2290

Johnston, A., Barton, M. J., Williams-Pritchard, G. A., \& Todorovic, M. (2018). YouTube for millenial nursing students: Using Internet technology to support student engagement with bioscience. Nurse Education in Practice, 31, 151-155. https://doi.org/10.1016/j.nepr.2018.06.002

ISSN 2313-7878. Hamut'ay 6(3). Setiembre-diciembre 2019. Págs. 12-25 
Kohli, C., Lancellotti, M., \& Thomas, S. (2017). Student attitudes toward hybrid business classes: Lessons for implementation Winter. Journal of the Academy of Business Education, 387-395.

Kum-Tien, D. T. (2019). Enhancing engineering students' community service experience through making YouTube videos. Journal of Engineering Science and Technology, 1, 44-55. Recuperado de https://bit.ly/2BKvN15

López-Belmonte, J., Pozo-Sánchez, S., Fuentes-Cabrera, A., Trujillo-Torres, J. M. (2019). Analytical competences of teachers in big data in the era of digitalized learning. Education Sciences, 9(177), 1-13. https://doi.org/10.3390/educsci9030177

Marsh, V. L., \& Hoff, M. J. (2019). New literacies in participatory cultures: The assumption of trust. E-Learning and Digital Media, 16(5), 393-412. https://doi. org/10.1177/2042753019863489

McAlister, R. B. (2014). Use of instructor-produced YouTube videos to supplement manual skills tranining in occupational therapy education. American Journal of Occupational Theraphy, 68, 67-72. https://doi.org/10.5014/ ajot.2014.685S04

Moghavvemi, S., Sulaiman, A., Ismawati-Jaafar, N., \& Kasem, N. (2018). Social media as a complementary learning tool for teaching and learning: The case of YouTube. The International Journal of Management Education, 16, 37-42. https://doi.org/10.1016/j.ijme.2017.12.001

Mohmed-Al-Sabaawi, M. Y., \& Mohamed-Dahlan, H. (2018). Acceptance model of social media for informal learning. In Saeed F., Gazem N., Patnaik S., Saed Balaid A., \& Mohammed F. (Eds.), Recent trends in information and communication technology (pp. 679-687). Malasya: Springer. https://doi.org/10.1007/978-3-319-59427-9_70

Orús, C., Barlés, M., Belanche, D., Casalo, L., Fraj, E., \& Gurrea, R. (2016). The effects of learner-generated videos for YouTube on learning outcomes and satisfaction. Computers \& Education, 95, 254-269. https://doi.org/10.1016/j.compedu.2016.01.007

Padilla-Partida, S. (2018). Usos y actitudes de los formadores de docentes ante las TIC. Entre lo recomendable y la realida de las aulas. Apertura, 10(1), 132-148. https://doi. org/10.32870/Ap.v10n1.1107

Picazo-Sánchez, L. (2016). Patrones del comportamiento viral. Documentación de las Ciencias de la Información, 39, 313-331. https://doi.org/10.5209/DCIN.54421

Pinzón-Capador, S. V., \& Guerrero-Nieto, C. H. (2018). Viviendo la práctica docente desde las dicotomías: La historia desde adentro cuando se implementan TIC en el salón de clase de inglés. HOW, 25(2), 69-89. https://doi. org/10.19183/how.25.2.459

Pöntinen, S., Dillon, P., \& Väisänen, P. (2017). Student teachers' discourse about digital technologies and transitions between formal and informal learning contexts. Education and Information Technologies, 22(1), 317-335. https://doi. org/10.1007/s10639-015-9450-0
Rodríguez-Gómez, D., Ion, G., Mercader, C., \& López-Crespo, S. (2019). Factors promoting informal and formal leraning strategies among school leaders. Studies in Continuing Education, 1-16. https://doi.org/10.1080/0158037X.2019.1600492

Sánchez-Vera, M. M., Solano-Fernández, I. M., \& González-Calatayud, V. (2016). Flipped-TIC: Una experiencia de flipped classroom con alumnos de Magisterio. RELATEC, 15(3), 69-81. https://doi.org/10.17398/1695288X.15.3.69

Sefton-Green, J. (2013). Learning at not-school: A review of study, theory, and advocacy for education in non-formal settings. Cambridge: MIT Press. https://doi.org/10.7551/ mitpress/9351.003.0002

Shoufan, A. (2018). Estimating the cognitive value of YouTube's educational videos: A learning analytics approach. Computer and Human Behavior, 92, 450-458. https://doi. org/10.1016/j.chb.2018.03.036

Silva-Pereira, J., Silva-Roque, J., \& Ferraz-Macário, L. (2018). Tecnologias na educação: Uma análise do uso de recursos tecnológicos por professores de uma instituição de ensino superior de Vitória da Conquista - BA. Humanidades \& Inovação, 5(9), 158-169.

Smith, R., \& Secoy, J. (2019). Exploring the music identity development of elementary education majors using ukelele and YouTube. Journal of Music Teacher Education, 29(1), 71-85. https://doi.org/10.1177/1057083719871026

Sorte, S. R., \& Rathod, S. B. (2016). Social networking sites as informal learning tool. Indian Journal of Physiology and Pharmacology, 60(4), 403-406. https://doi.org/10.1108/ TLO-10-2014-0058

Stephen, C., \& Plowman, L. (2003). Information and communication technologies in pre-school settings: A review of the literature. International Journal of Early Years Education, 11(3), 223-234. https://doi. org/10.1080/0966976032000147343

Sun, K., Wang, G., \& Liu, M. (2017). Stop-motion para la alfabetización digital en Educación Primaria. Comunicar, 51, 93-103. https://doi.org/10.3916/C51-2017-09

Tan, E. (2013). Informal learning on YouTube: Exploring digital literacy in independent online learning. Learning Media and Technology, 38(4), 463-477. https://doi.org/10. 1080/17439884.2013.783594

Toolabi, K., Parsaei, R., Elyasinia, F., \& Zamanian, A. (2019). Reliability and educational value of laparoscopic sleeve gastrectomy surgery videos on YouTube. Obesity Surgery, 29(9), 2806-2813. https://doi.org/10.1007/s11695019-03907-3

Tuzel, S., \& Hobbs, R. (2017). El uso de las redes sociales y la cultura popular para una mejor comprensión intercultural. Comunicar, 25, 63-72. https://doi.org/10.3916/C512017-06

Van-den-Eynde, J., Crauwels, A., George-Demaerel, P., Van-Eycken, L., Bullens, D. ...Toelen, J. (2019). YouTube videos as a source of information about immunology for medical students: Cross-sectional study. JMIR Med Educ, 
Del aula a la escuela en línea universal: Dimensiones temáticas en canales de Youtube

5(1), 1-14. https://doi.org/10.2196/12605

Vargas-Vergara, M. (2019). Temas para debate. Nuevas tecnologías para el aprendizaje y la formación de una sociedad en red: Salvando la tercera brecha digital. Aularia, 8(2), 73108. Recuperado de https://bit.ly/2C4wi6F

Vizcaíno-Verdú, A., Contreras-Pulido, P., \& Guzmán-Franco, M. D. (2019). Lectura y aprendizaje informal en YouTube: El booktuber. Comunicar, 59, 95-104. https://doi. org/10.3916/C59-2019-09 
Anexo

\begin{tabular}{|c|c|c|c|}
\hline $\mathrm{N}^{0}$ & Temática inicial & $\begin{array}{c}\text { Frecuencia } \\
\text { de temáticas } \\
\text { en canales }\end{array}$ & $\begin{array}{l}\% \text { equiva- } \\
\text { lente }\end{array}$ \\
\hline 1 & Curiosidades & 80 & 18 \\
\hline 2 & Física & 74 & 17 \\
\hline 3 & Matemáticas & 72 & 16 \\
\hline 4 & Lengua inglesa & 40 & 9 \\
\hline 5 & Historia & 17 & 4 \\
\hline 6 & Biología & 16 & 4 \\
\hline 7 & Química & 15 & 3 \\
\hline 8 & Astronomía & 14 & 3 \\
\hline 9 & Informática & 9 & 2 \\
\hline 10 & Economía & 5 & 1 \\
\hline 11 & Tecnología & 5 & 1 \\
\hline 12 & Música & 5 & 1 \\
\hline 13 & TIC & 5 & 1 \\
\hline 14 & Naturaleza & 5 & 1 \\
\hline 15 & Medioambiente & 5 & 1 \\
\hline 16 & Educación infantil & 4 & 1 \\
\hline 17 & Política & 4 & 1 \\
\hline 18 & Psicología & 4 & 1 \\
\hline 19 & Finanzas & 3 & 1 \\
\hline 20 & Antropología & 3 & 1 \\
\hline 21 & Animales & 3 & 1 \\
\hline 22 & Fauna & 3 & 1 \\
\hline 23 & Medicina & 3 & 1 \\
\hline 24 & Salud & 3 & 0 \\
\hline 25 & Filosofía & 2 & 0 \\
\hline 26 & Genética & 2 & 0 \\
\hline 27 & Arqueología & 2 & 0 \\
\hline 28 & Veterinaria & 2 & 0 \\
\hline 29 & Meteorología & 2 & 0 \\
\hline 30 & $\begin{array}{l}\text { Ingeniería multidisci- } \\
\text { plinar }\end{array}$ & 2 & 0 \\
\hline
\end{tabular}

\begin{tabular}{|c|c|c|c|}
\hline $\mathrm{N}^{0}$ & Temática inicial & $\begin{array}{c}\text { Frecuencia } \\
\text { de temáticas } \\
\text { en canales }\end{array}$ & $\begin{array}{l}\% \text { equiva- } \\
\text { lente }\end{array}$ \\
\hline 31 & Geografía & 2 & 0 \\
\hline 32 & Videojuegos & 2 & 0 \\
\hline 33 & Biodiverisdad & 2 & 0 \\
\hline 34 & Arte & 2 & 0 \\
\hline 35 & Deporte & 2 & 0 \\
\hline 36 & Farmacología & 2 & 0 \\
\hline 37 & Enfermería & 2 & 0 \\
\hline 38 & Geología & 2 & 0 \\
\hline 39 & Anatomía & 2 & 0 \\
\hline 40 & Robótica & 1 & 0 \\
\hline 41 & Agricultura & 1 & 0 \\
\hline 42 & Historia del arte & 1 & 0 \\
\hline 43 & Ingeniería industrial & 1 & 0 \\
\hline 44 & Ingeniería mecánica & 1 & 0 \\
\hline 45 & Inteligencia artificial & 1 & 0 \\
\hline 46 & Odontología & 1 & 0 \\
\hline 47 & Lengua árabe & 1 & 0 \\
\hline 48 & Biotecnología & 1 & 0 \\
\hline 49 & Nutrición & 1 & 0 \\
\hline 50 & Astrología & 1 & 0 \\
\hline 51 & Electrónica & 1 & 0 \\
\hline 52 & Pedagogía & 1 & 0 \\
\hline 53 & Lengua japonesa & 1 & 0 \\
\hline 54 & Turismo & 1 & 0 \\
\hline 55 & Teología & 1 & 0 \\
\hline 56 & Mitología & 1 & 0 \\
\hline 57 & Literatura & 1 & 0 \\
\hline 58 & Ecología & 1 & 0 \\
\hline 59 & Energías renovables & 1 & 0 \\
\hline
\end{tabular}

Fuente: Elaboración propia (2019). 